\title{
Universal transforms of the geometric series under generalized Riesz methods
}

\author{
L. Bernal-González, M.C. Calderón-Moreno* and W. Luh
}

Dedicated to the memory of Dieter Gaier

\begin{abstract}
In this paper generalized Riesz methods $(R, p, M)$ of summability are considered. We prove that, to each open set $O \subset \mathbb{C}$ with adequate topological properties and each sequence $\left\{P_{n}\right\} \subset \mathbb{C}$ tending to infinity, we can associate a corresponding P-regular $(R, p, M)$-method so that the geometric series and a certain trigonometric series become universal in the sense that its $(R, p, M)$-transforms approximate any member of certain spaces of holomorphic functions or measurable functions.
\end{abstract}

2000 Mathematics Subject Classification: Primary 30E10. Secondary 40C05, 40G99, 42A10.

Key words and phrases: Riesz method, universal function, geometric series, trigonometric series, P-regularity.

\section{Introduction}

Suppose that $p:=\left\{p_{\nu}\right\}_{\nu=0}^{\infty}$ is a sequence of complex numbers with the property that

$$
P_{n}:=\sum_{\nu=0}^{m_{n}} p_{\nu} \neq 0 \quad\left(n \in \mathbb{N}_{0}\right)
$$

*The first two authors have been partially supported by Plan Andaluz de Investigación de la Junta de Andalucía. 
for a subsequence $M:=\left\{m_{n}\right\}_{n=0}^{\infty}$ of $\mathbb{N}_{0}:=\mathbb{N} \cup\{0\}=\{0,1,2, \ldots\}$. The row-finite matrix $A=\left[\alpha_{n \nu}\right]$ with entries

$$
\alpha_{n \nu}:=\frac{p_{\nu}}{P_{n}} \text { for } 0 \leq \nu \leq m_{n} ; \quad \alpha_{n \nu}:=0 \text { for } \nu>m_{n}
$$

generates a summability method "of weighted mean type", which occasionally is denoted as a generalized Riesz method $(R, p, M)$ and which was first investigated by Faulstich (see [2]). Such a method $(R, p, M)$ is regular (by the well known Silverman-Toeplitz conditions) if and only if

$$
\lim _{n \rightarrow \infty} P_{n}=\infty, \quad \sup _{n} \frac{1}{\left|P_{n}\right|} \sum_{\nu=0}^{m_{n}}\left|p_{\nu}\right|<\infty ;
$$

it is P-regular if and only if

$$
\lim _{n \rightarrow \infty} P_{n}=\infty, \sup _{n} \frac{1}{\left|P_{n}\right|} \sum_{\nu=0}^{m_{n}}\left|p_{\nu}\right| \rho^{\nu}<\infty \text { for all } \rho \in(0,1)
$$

(see Remark 3.2 (2) below).

We recall that if $A=\left[\alpha_{n \nu}\right]_{n, \nu=0}^{\infty}$ is a general infinite matrix with complex entries, then $A$ (or the summability method generated by it) is called regular if it preserves convergence of limits of sequences, that is, given a sequence $\left\{s_{n}\right\}$ with $s_{n} \rightarrow s \in \mathbb{C}$ then it is also $A$-summable to $s$ or, in other words, the sequence $\sigma_{n}:=\sum_{\nu=0}^{\infty} \alpha_{n \nu} s_{\nu}$ of its $A$-transforms also converges to $s$. And $A$ is called P-regular ("regular for power series") whenever for any given power series $f(z)=\sum_{\nu=0}^{\infty} a_{\nu} z^{\nu}$ with radius of convergence $R \in(0, \infty)$ the sequence $\sigma_{n}(z):=\sum_{\nu=0}^{\infty} \alpha_{n \nu} s_{\nu}(z)$ of its $A$-transforms (where $s_{\nu}(z)=\sum_{\mu=0}^{\nu} a_{\mu} z^{\mu}$ ) converges to $f(z)$ compactly in $\{z:|z|<R\}$. The exact conditions for regularity and P-regularity of a matrix $A$ are due respectively to Silverman and Toeplitz (see for instance [15] or [17, pages 6-7]) and Luh (see [8]).

In 1945 Mensǒv [12] proved the existence of a so-called universal trigonometric series

$$
\sum_{\nu=0}^{\infty}\left\{a_{\nu} \cos \nu t+b_{\nu} \sin \nu t\right\}
$$

with the property that for every Lebesgue measurable function $\varphi$ on $[0,2 \pi]$ there exists a subsequence $\left\{n_{k}\right\}$ of the natural numbers such that the corresponding sequence of partial sums

$$
s_{n_{k}}(t)=\sum_{\nu=0}^{n_{k}}\left\{a_{\nu} \cos \nu t+b_{\nu} \sin \nu t\right\}
$$




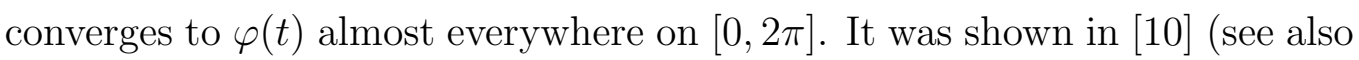
[13]) that there exist universal Taylor series $\sum_{\nu=0}^{\infty} a_{\nu} z^{\nu}$ with radius of convergence 1 which for $z=e^{i t} \in \mathbb{D}$ become universal in the sense of Mensǒv.

The trigonometric series $\sum_{\nu=0}^{\infty}\{\cos \nu t+\sin \nu t\}$ or the geometric series $\sum_{\nu=0}^{\infty} z^{\nu}$ obviously cannot have corresponding universal properties. However it is the aim of the present paper to apply $(R, p, M)$-methods to these series, in such a way that they become universal in the sense that the corresponding transforms approximate any member of certain spaces of holomorphic and measurable functions. Our results strongly generalize those, which were obtained in $[3]$.

The outline of the paper is as follows. Section 2 is auxiliary and in it a topological, crucial property is considered in order to be used later. In Section 3 we present two results on approximation of holomorphic functions. Section 4 is again auxiliary, and in it a general statement on Radon measures is shown; this section is of independent interest. In Section 5 we employ the assertions of the foregoing sections to establish a strong result about approximation of Lebesgue-measurable functions.

\section{Sets with an exhausting property}

Throughout this paper we use the following notations and abbreviations.

For an open set $O \subset \mathbb{C}$ we denote by $H(O)$ as usual the family of all functions which are holomorphic in $O$. If $K \subset \mathbb{C}$ is a compact set then $A(K)$ stands for the collection of all functions which are continuous on $K$ and holomorphic in the interior $K^{0}$ of $K$. By $\Longrightarrow$ we denote uniform convergence on a set $A \subset \mathbb{C}$, while $\Rightarrow$ stands for uniform convergence on any compact subset of $A$. Finally, $\mathbb{D}$ will represent the open unit disk $\{z:|z|<$ $1\}$.

By $\mathcal{M}$ we denote the collection of all compact sets $K$ of the complex plane $\mathbb{C}$ which have connected complement $K^{c}$.

Definition 2.1. Suppose that $F$ is a set in $\mathbb{C}$. Then $F$ has the property $E$ 
("exhausting property") if either $F=\emptyset$, or $F \neq \emptyset$ and there exists a sequence $\left\{K_{n}\right\} \subset \mathcal{M}$ with $K_{n} \subset F$ for all $n \in \mathbb{N}$ such that for any $K \subset F$ with $K \in \mathcal{M}$ there exists an $n_{0}=n_{0}(K) \in \mathbb{N}$ with $K \subset K_{n_{0}}$. Any such a sequence $\left\{K_{n}\right\}$ is called an "exhausting sequence" for $F$.

Examples 2.2. Let $G \subset \mathbb{C}$ be a simply connected domain, then $G$ has the property $E$. Indeed, if $G=\mathbb{C}$ we choose $S^{(n)}:=\{z:|z| \leq n\}$, and in the case that $G \neq \mathbb{C}$ let $\phi$ be a conformal mapping of $\mathbb{D}$ onto $G$ and consider the sets

$$
S^{(n)}:=\phi\left(\left\{z:|z| \leq 1-\frac{1}{n+1}\right\}\right) \quad(n \in \mathbb{N}) .
$$

Then in both cases $\left\{S^{(n)}\right\}_{n=1}^{\infty}$ is an exhausting sequence for $G$ (actually for any compact set $K \subset G$ there exists an $n_{0} \in \mathbb{N}$ with $\left.K \subset S^{\left(n_{0}\right)}\right)$.

More generally any domain $G \subset \mathbb{C}$ has the property $E$. In fact it is well known that any open set $G \subset \mathbb{C}$ has this exhausting property (cf. [9, page 198], [5, chapter 2.2]). The referee has kindly supplied an alternative proof of this assertion, which is a modification of the proof of Lemma 2.1 in [10] (see also [11]): In [10] having a locally finite number of components we succeds to consider a finite subset of $\mathbb{Q}+i \mathbb{Q}$. In the present situation the intersection of the component of $G$ with a big closed disk is compact and is at a positive distance $\delta$ from a compact set $K$. By compactness we can find a finite number of open disks with centers in this intersection, all with radii $\frac{\delta}{1000}$, which cover this intersection. We can select an element of $\mathbb{Q}+i \mathbb{Q}$ in each one of these open disks. Then we arrive again to deal with a finite subset of $\mathbb{Q}+i \mathbb{Q}$ and the approach of [10, Lemma 2.1] applies.

The following examples show that closed sets may or may not have the property $E$.

Examples 2.3. 1. If $O \subset \mathbb{C}$ is an open set with infinitely many components then $F=O^{c}$ has in general not the property E. This can be seen for instance by the example (see [10])

$$
O=\bigcup_{n=1}^{\infty}\left\{z:\left|z-2^{-n}\right|<2^{-n-2}\right\} .
$$


However, if it is supposed that the number of components of $O$ is locally finite -that is, every compact subset $L \subset \mathbb{C}$ only intersects a finite number $N=N(L)$ of components of $O$ - then it was shown by Melas and Nestoridis [10, Lemma 2.1] that $O^{c}$ has the property E.

2. In particular, if $O \subset \mathbb{C}$ is an open set with a finite number of components, then $F=O^{c}$ has the property E.

3. Consequently, if $G$ is any domain in $\mathbb{C}(=$ a nonempty connected open subset of $\mathbb{C}$ ), then $F=G^{c}$ has the property E. In this case, we can provide with the following easy proof, which is independent of that of [10]: The result is clear if $G=\mathbb{C}$. Suppose that $G \neq \mathbb{C}$ and choose a closed circle $S=\left\{z:\left|z-z_{0}\right| \leq r\right\} \subset G$. Let $\left\{L_{n}\right\}$ be an enumeration of all Jordan domains in $S^{c}$ which are bounded by polygons with vertices in points which have rational real and imaginary parts. Then each closure $\overline{L_{n}}$ belongs to $\mathcal{M}$ and for any set $L \in \mathcal{M}$ with $L \subset S^{c}$ there exists an $n_{0}$ with $L \subset \overline{L_{n_{0}}}$. It is easy to see that the sequence $\left\{K_{n}\right\}$ with $K_{n}:=\overline{L_{n}} \cap G^{c}$ is an exhausting sequence for $G^{c}$.

\section{Approximation of holomorphic functions}

We first proof the following results.

Theorem 3.1. Let be prescribed:

- an open set $O \subset \mathbb{C}$ with simply connected components and $\mathbb{D} \subset O$, $1 \notin O$;

- a set $F \subset O^{c}$ which has the property E;

- a function $\varphi \in H(O)$ with $\left.\varphi\right|_{\mathbb{D}}=\varphi_{0}$, where $\varphi_{0}(z) \equiv 0$;

- a sequence $\left\{P_{n}\right\} \subset \mathbb{C} \backslash\{0\}$ with $P_{n} \rightarrow \infty$.

(a) Then there exist sequences $\left\{p_{\nu}\right\} \subset \mathbb{C}$ and $\left\{m_{n}\right\} \subset \mathbb{N}_{0}$ such that $P_{n}=$ $\sum_{\nu=0}^{m_{n}} p_{\nu}(n \in \mathbb{N})$ and

$$
\tau_{n}(z):=\frac{1}{P_{n}} \sum_{\nu=0}^{m_{n}} p_{\nu} z^{\nu} \Rightarrow \varphi(z) \quad(n \rightarrow \infty) .
$$


(b) If in addition any set $K \in \mathcal{M}, K \subset F, 1 \notin K$ and any function $f \in A(K)$ are given then there exists a sequence $\left\{n_{k}\right\}$ with

$$
\tau_{n_{k}}(z) \underset{K}{\Longrightarrow} f(z) \quad(k \rightarrow \infty)
$$

Proof. 1. Suppose that $O=\bigcup_{\nu \in I} G_{\nu}$, where $0 \in I$ and $I \subset \mathbb{N}_{0}$, that the $G_{\nu}$ 's

are pairwise disjoint simply connected domains (the components of $O$ ) and assume $\mathbb{D} \subset G_{0}$.

For $\nu \in I$ choose a conformal mapping $\phi_{\nu}$ of $\mathbb{D}$ onto $G_{\nu}$ and consider the sets

$$
\begin{gathered}
S_{\nu}^{(n)}:=\phi_{\nu}\left(\left\{z:|z| \leq 1-\frac{1}{n+1}\right\}\right) \quad(n \in \mathbb{N}, \nu \in I), \\
T_{n}:=S_{0}^{(n)}, S_{n}:=\bigcup_{\substack{\nu \in I \\
1 \leq \nu \leq n}} S_{\nu}^{(n)} \quad(n \in \mathbb{N}),
\end{gathered}
$$

which are compact, have connected complement and the sets $T_{n}$ and $S_{n}$ are pairwise disjoint for each $n \in \mathbb{N}$.

Since $F$ has the property $E$ it is not hard to see that also $F_{1}:=F \backslash\{1\}$ has the property $E$. Without loss of generality we may assume that $F_{1} \neq \phi$.

Let $\left\{K_{n}^{*}\right\}$ be an exhausting sequence for $F_{1}$ and denote by $\left\{\Pi_{n}^{*}\right\}$ an enumeration of all polynomials whose coefficients have rational real and imaginary parts. Finally let $\left\{\left(K_{n}, \Pi_{n}\right)\right\}_{n=1}^{\infty}$ be an arrangement of the sets $K_{n}^{*}$ and the polynomials $\Pi_{n}^{*}$ in which any combination $\left(K_{r}^{*}, \Pi_{s}^{*}\right)$ occurs infinitely often. It follows that for each $n$ the sets $T_{n}, S_{n}, K_{n},\{1\}$ are pairwise disjoint.

2. We construct by induction a sequence $\left\{Q_{n}\right\}$ of polynomials and sequences $\left\{q_{n}\right\}$ and $\left\{m_{n}\right\}$ of nonnegative integers.

Suppose that $Q_{0}(z) \equiv P_{0}$ and $q_{0}=0$ and assume that for an $n \in \mathbb{N}$ the polynomials $Q_{0}, \ldots, Q_{n-1}$ and the numbers $q_{0}, \ldots, q_{n-1}$ have already been determined. The degree of the polynomial $z^{q_{n-1}} Q_{n-1}(z)$ will be denoted by $m_{n-1}$.

We choose $q_{n} \in \mathbb{N}$ so great that $q_{n}>m_{n-1}$ and using Runge's approximation theorem (see $\left[4\right.$, Chapter II,3]) we find a polynomial $Q_{n}$ which satisfies simultaneously

$$
Q_{n}(1)=P_{n}-P_{n-1}
$$




$$
\begin{gathered}
\max _{T_{n}}\left|Q_{n}(z)\right|<\frac{1}{n^{2} \max _{T_{n}}\left|z^{q_{n}}\right|}, \\
\max _{S_{n}}\left|Q_{n}(z)-\frac{P_{n} \cdot \varphi(z)-\sum_{\nu=0}^{n-1} z^{q_{\nu}} Q_{\nu}(z)}{z^{q_{n}}}\right|<\frac{1}{\max _{S_{n}}\left|z^{q_{n}}\right|}, \\
\max _{K_{n}}\left|Q_{n}(z)-\frac{P_{n} \cdot \prod_{n}(z)-\sum_{\nu=0}^{n-1} z^{q_{\nu}} Q_{\nu}(z)}{z^{q_{n}}}\right|<\frac{1}{\max _{K_{n}}\left|z^{q_{n}}\right|} .
\end{gathered}
$$

By induction we get $\left\{Q_{n}\right\},\left\{q_{n}\right\},\left\{m_{n}\right\}$; note that $m_{n}>m_{n-1}$ and $q_{n}>q_{n-1}$ for all $n \in \mathbb{N}$.

3. The series $\sum_{\nu=0}^{\infty} z^{q_{\nu}} Q_{\nu}(z)$ converges by (2) compactly in $G_{0}$ and therefore $g(z):=\sum_{\nu=0}^{\infty} z^{q_{\nu}} Q_{\nu}(z)$ is holomorphic in $G_{0}$. The properties of the $q_{n}$ and $m_{n}$ imply that the polynomials $z^{q_{n-1}} Q_{n-1}(z)$ and $z^{q_{n}} Q_{n}(z)$ do not have powers in common. Therefore, if the power series of the function $g$ around the origin is denoted by $g(z)=\sum_{\nu=0}^{\infty} p_{\nu} z^{\nu}$, we obtain

$$
\sum_{\nu=0}^{m_{n}} p_{\nu} z^{\nu}=\sum_{\nu=0}^{n} z^{q_{\nu}} Q_{\nu}(z)
$$

¿From (1) we get $\sum_{\nu=0}^{m_{n}} p_{\nu}=\sum_{\nu=0}^{n} Q_{\nu}(1)=P_{n} \rightarrow \infty$ for $n \rightarrow \infty$ which implies that the power series $\sum_{\nu=0}^{\infty} p_{\nu} z^{\nu}$ has radius of convergence 1 . We obviously have $\varphi_{\mid G_{0}}(z) \equiv 0$, which together with (2) gives

$$
\tau_{n}(z):=\frac{1}{P_{n}} \sum_{\nu=0}^{m_{n}} p_{\nu} z^{\nu}=\frac{1}{P_{n}} \sum_{\nu=0}^{n} z^{q_{\nu}} Q_{\nu}(z) \overline{\bar{G}}_{0} \varphi_{\mid G_{0}}(z) \equiv 0 .
$$

4. The property (3) implies

$$
\max _{S_{n}}\left|\tau_{n}(z)-\varphi(z)\right|=\max _{S_{n}}\left|\frac{1}{P_{n}} \sum_{\nu=0}^{n} z^{q_{\nu}} Q_{\nu}(z)-\varphi(z)\right|<\frac{1}{\left|P_{n}\right|}
$$

and we obtain $\tau_{n}(z) \overline{\bar{G}}_{\nu} \varphi(z)$ for all $\nu \in I, \nu>0$. Together with step 3 we have $\tau_{n}(z) \equiv \varphi(z)$ 
5. From (4) we get

$$
\max _{K_{n}}\left|\tau_{n}(z)-\Pi_{n}(z)\right|<\frac{1}{\left|P_{n}\right|}
$$

Let now be given a set $K \in \mathcal{M}$ with $K \subset F \backslash\{1\}=F_{1}$ and a function $f \in A(K)$. Then by Mergelian's theorem (see [4, Chapter III,2]) there exists a sequence $\left\{s_{k}\right\}$ with $s_{k} \rightarrow \infty$ and $\Pi_{s_{k}}^{*}(z) \underset{\bar{K}}{\Longrightarrow} f(z)$. By the exhaustion property of $F_{1}$ there exists an $r_{0}$ with $K \subset K_{r_{0}}^{*}$ and we find a sequence $\left\{n_{k}\right\}$ with $\Pi_{n_{k}}(z)=\Pi_{s_{k}}^{*}(z)$ and $K_{n_{k}}=K_{r_{0}}^{*}$ for all $k$. Together with (5) we get

$$
\tau_{n_{k}}(z) \underset{K}{\Longrightarrow} f(z)
$$

which proves the theorem.

Remarks 3.2. 1. We consider especially the open set $O=\mathbb{D}$. By the Example 2.3(3) the set $F:=\mathbb{D}^{c}$ has the property E. Therefore Theorem 3.1 generalizes Theorem 1 of [1], where we proved the same approximation properties given in the former theorem but just in the very special case $O=\mathbb{D}, F=\mathbb{D}^{c}, \varphi=0, K \subset \partial \mathbb{D} \backslash\{1\}$. Several consequences on the approximation of measurable functions by universal trigonometric series are given in [1]. This will also be treated later in our current, more general situation, see Section 5 .

2. The sequence $p=\left\{p_{\nu}\right\}$ which was constructed in the proof of Theorem 3.1 obviously satisfies $\tau_{n}(1)=1$ for all $n \in \mathbb{N}_{0}$ and $\tau_{n}(z) \Rightarrow 0$. It is not difficult to show that these conditions are equivalent to those which were mentioned in the introduction for $P$-regularity (for details we refer to [8]). Therefore the considered $(R, p, M)$-method is $P$-regular.

3 . However it is easy to see that the method $(R, p, M)$ in general cannot chosen to be regular. Indeed, suppose that the sets $O$ and $F$ have the property that there exists a $\varsigma_{0} \in \partial \mathbb{D} \cap F$ with $\varsigma_{0} \neq 1$. If $(R, p, M)$ would be regular, then there would exist a constant $C$ with

$$
\frac{1}{\left|P_{n}\right|} \sum_{\nu=0}^{m_{n}}\left|p_{\nu}\right| \leq C \quad \text { for all } n \in \mathbb{N} \text {. }
$$


On the other hand by property (b) of Theorem 3.1 we can find a sequence $\left\{n_{k}\right\}$ of natural numbers with

$$
\tau_{n_{k}}\left(\varsigma_{0}\right)=\frac{1}{P_{n_{k}}} \sum_{\nu=0}^{m_{n_{k}}} p_{\nu} \varsigma_{0}^{\nu} \rightarrow C+1 \quad \text { for } k \rightarrow \infty
$$

which is obviously not possible.

¿From Theorem 3.1 the following statement about the universal behaviour of the $(R, p, M)$-transforms of the geometric series follows very easily.

Theorem 3.3. Suppose that $O, F$ and $\left\{P_{n}\right\}$ are the same as in Theorem 3.1. Let be given a function $\Phi \in H(O)$ with $\left.\Phi\right|_{\mathbb{D}}=\Phi_{0}$, where $\Phi_{0}(z) \equiv \frac{1}{1-z}$.

(a) Then there exist sequences $\left\{p_{\nu}\right\} \subset \mathbb{C}$ and $\left\{m_{n}\right\} \subset \mathbb{N}_{0}$ such that $P_{n}=$ $\sum_{\nu=0}^{m_{n}} p_{\nu}(n \in \mathbb{N})$ and

$$
\sigma_{n}(z):=\frac{1}{P_{n}} \sum_{\nu=0}^{m_{n}} p_{\nu} \sum_{\mu=0}^{\nu} z^{\mu} \equiv \Phi(z) .
$$

(b) If in addition any set $K \in \mathcal{M}, K \subset F, 1 \notin K$ and any function $f \in A(K)$ are given then there exists a sequence $\left\{n_{k}\right\}$ with

$$
\sigma_{n_{k}}(z) \underset{K}{\Longrightarrow} f(z)
$$

Proof. Let $\left\{\tau_{n}(z)\right\}$ be the sequence which by Theorem 3.1 exists according to $O, F,\left\{P_{n}\right\}$ and the function $\varphi$ with

$$
\varphi(z):= \begin{cases}0 & \text { if } z \in G_{0} \\ \frac{1}{z}-\frac{1-z}{z} \Phi(z) & \text { if } z \in O \backslash G_{0} .\end{cases}
$$

Then there are sequences $\left\{p_{\nu}\right\}$ and $\left\{m_{n}\right\}$ such that

$$
\tau_{n}(z):=\frac{1}{P_{n}} \sum_{\nu=0}^{m_{n}} p_{\nu} z^{\nu} \Rightarrow \varphi(z) .
$$


For $z \neq 1$ we obtain

$$
\sigma_{n}(z)=\frac{1}{P_{n}} \sum_{\nu=0}^{m_{n}} p_{\nu} \sum_{\mu=0}^{\nu} z^{\mu}=\frac{1}{1-z}-\frac{z}{1-z} \tau_{n}(z),
$$

which implies

$$
\sigma_{n}(z) \equiv \Phi(z)
$$

If a set $K \in \mathcal{M}, K \subset F, 1 \notin K$ and a function $f \in A(K)$ are given then there exists a sequence $\left\{n_{k}\right\}$ such that

$$
\tau_{n_{k}}(z) \underset{K}{z} \frac{1}{z}-\frac{1-z}{z} f(z)
$$

which implies $\sigma_{n_{k}}(z) \underset{K}{\Longrightarrow} f(z)$.

\section{An approximation lemma}

In this section an auxiliary result on the approximation of measurable functions will be proved, see Lemma 4.1. Although it will be applied on measurable functions on $\mathbb{C}$-specifically, in Theorem 5.1 - we prefer to state it in some generality, because this does not cause much more difficulty. Moreover, we believe that the lemma could be of independent interest. Nevertheless, a number of concepts and assertions from measure theory are needed. We recall that, for a positive measure $\mu$ on some $\sigma$-algebra $\Sigma$ of subsets of a set $X$, a subset $A \in \Sigma$ is called $\sigma$-finite if there are countable many sets $A_{n} \in \Sigma$ $(n \in \mathbb{N})$ satisfying $\mu\left(A_{n}\right)<\infty(n \in \mathbb{N})$ and $A=\bigcup_{n=1}^{\infty} A_{n}$. The measure $\mu$ is $\sigma$-finite whenever $X$ is $\sigma$-finite itself, hence every measurable set is also $\sigma$-finite in this case. The measure $\mu$ is complete if $[A \in \Sigma, B \subset A, \mu(A)=0]$ implies $B \in \Sigma$.

For the sake of convenience we introduce the following concept. If $X$ is a topological space and $\mu$ is a measure on a $\sigma$-algebra of subsets of $X$ containing the collection $\mathcal{B}$ of Borel sets of $X$ (that is, $\mathcal{B}$ is the smallest $\sigma$-algebra on $X$ that contains the open sets of $X$ ), then we say that $X$ is thin-connected (with respect to $\mu$ ) whenever the following property holds: 
For prescribed $\varepsilon>0$ and points $a, b \in X$, there exists a connected open set $U$ in $X$ containing $a, b$ such that $\mu(U)<\varepsilon$.

Let $X$ be a locally compact Hausdorff space and suppose that $\Sigma$ is a $\sigma$ algebra of subsets of $X$ containing $\mathcal{B}$. Then a measure $\mu$ on $\Sigma$ is said to be regular (see [14, Chapter 22]) or a Radon measure provided that

(i) $\mu(C)<\infty$ for all compact subsets $C$ of $X$,

(ii) $\mu$ is externally regular, that is, $\mu(A)=\inf \{\mu(U): U$ is an open set in $X$ with $A \subset U\}$ for each set $A \in \Sigma$, and

(iii) $\mu(U)=\sup \{\mu(C): C$ is a compact set in $X$ with $C \subset U\}$ for each open set $U$ of $X$.

We denote by $\mathbb{K}$ any of the fields $\mathbb{R}, \mathbb{C}$. If $L$ is a compact set, then $C(L)$ will stand, as usual, for the space of the continuous $\mathbb{K}$-valued functions on $L$, endowed with the maximum norm.

Lemma 4.1. Assume that $X$ is a Hausdorff locally compact space and that $\mu$ is a measure on some $\sigma$-algebra $\Sigma$ of subsets of $X$ with $\mathcal{B} \subset \Sigma$. Suppose that $Y \in \Sigma$ and that $f: Y \rightarrow \mathbb{K}$ is a measurable function on $Y$. Let $\mathcal{A}$ be a family of $\mathbb{K}$-valued continuous functions on $X$. Assume, in addition, that the following conditions hold:

(a) The measure $\mu$ is regular, complete and $\sigma$-finite.

(b) There exists a dense subset $D$ in $X$ with $D \in \Sigma$ and $\mu(D)=0$.

(c) Every open subset of $X$ has at most countably many components.

(d) The space $X$ is thin-connected.

(e) For every compact set $L \subset Y$ with empty interior and connected complement $X \backslash L$ the (collection of restrictions to $L$ of the members of the) family $\mathcal{A}$ is dense in $C(L)$.

Then there is a sequence $\left\{f_{n}\right\}_{n=1}^{\infty} \subset \mathcal{A}$ such that $f_{n} \rightarrow f(n \rightarrow \infty)$ almost everywhere on $Y$. 
Proof. Since $\mu$ is $\sigma$-finite (by (a)), there are countable many sets $A_{n} \in \Sigma$ $(n \in \mathbb{N})$ with $\mu\left(A_{n}\right)<\infty$ such that $Y=\bigcup_{n=1}^{\infty} A_{n}$. Replacing $A_{n}$ by $A_{1} \cup \cdots \cup A_{n}$ if necessary, it may be assumed that the sequence $\left\{A_{n}\right\}_{n=1}^{\infty}$ is increasing.

¿From (b), there is a dense measurable set $D$ in $X$ with $\mu(D)=0$. Then the sets $B_{n}:=A_{n} \backslash D(n \in \mathbb{N})$ satisfy $B_{n} \subset A_{n}, \mu\left(B_{n}\right)=\mu\left(A_{n}\right)<\infty$ and $B_{n}^{0}=\emptyset$.

Since $\mu$ is regular and $\sigma$-finite, it is also internally regular for all measurable sets (see for instance [14, Proposition 22.5]), that is, condition (iii) of the definition of Radon measure holds in fact for any $A \in \Sigma$, in particular for every $A \in \Sigma$ with $\mu(A)<\infty$. Recall that $\mu$ is also complete. In this situation we can apply Lusin's theorem (in the form stated, for instance, in [16, Chapter 2]), yielding the existence of continuous functions $g_{n}: X \rightarrow \mathbb{K}$ $(n \in \mathbb{N})$ such that

$$
\mu\left(\left\{x \in X: h_{n}(x) \neq g_{n}(x)\right\}<\frac{1}{2^{n}} \quad(n \in \mathbb{N}),\right.
$$

where each function $h_{n}: X \rightarrow \mathbb{K}$ is defined as

$$
h_{n}(x)= \begin{cases}f(x) & \text { if } x \in B_{n} \\ 0 & \text { otherwise }\end{cases}
$$

Now we define $C_{n}:=\left\{x \in B_{n}: h_{n}(x)=g_{n}(x)\right\}(n \in \mathbb{N})$. Obviously, the restriction of $h_{n}$ to $C_{n}$ is continuous. Observe that by (6) we have $\mu\left(B_{n} \backslash\right.$ $\left.C_{n}\right)<1 / 2^{n}$. From internal regularity, there is for each $n \in \mathbb{N}$ a compact set $D_{n} \subset C_{n}$ with $\mu\left(C_{n} \backslash D_{n}\right)<1 / 2^{n}$.

The following step is to apply properties (c)-(d). For every $n \in \mathbb{N}$, consider the collection $\left\{E_{n, j}\right\}_{j \in I(n)}$ of the connected components of the open set $X \backslash D_{n}$, where each $I(n)$ is either $\mathbb{N}$ or a finite set $\{1,2, \ldots, N(n)\}$. Fix $n \in \mathbb{N}$ and assume, for a moment, that $X \backslash D_{n}$ is not connected. Now fix points $x_{1}^{(n)} \in E_{n, 1}, x_{2}^{(n)} \in E_{n, 2}, \ldots$ and select, by thin-connectedness, connected open subsets $O_{n, k} \subset X(k \geq 2)$ such that $\left\{x_{1}^{(n)}, x_{k}^{(n)}\right\} \subset O_{n, k}$ and $\mu\left(O_{n, k}\right)<1 / 2^{n+k}$. Define the sets $U_{n}(n \in \mathbb{N})$ by

$$
U_{n}= \begin{cases}\emptyset & \text { if } X \backslash D_{n} \text { is connected } \\ \bigcup_{k \in I(n) \backslash\{1\}} O_{n, k} & \text { otherwise. }\end{cases}
$$

Then each $U_{n}$ is a connected open set and $\mu\left(U_{n}\right)<1 / 2^{n}$. Furthermore, the sets $L_{n}:=D_{n} \backslash U_{n}(n \in \mathbb{N})$ satisfy the following properties: 
- they are compact, because $D_{n}$ is compact and $U_{n}$ is open,

- $L_{n} \subset Y$, because $L_{n} \subset D_{n} \subset C_{n} \subset B_{n} \subset A_{n} \subset Y$,

- $L_{n}^{0}=\emptyset$, because $B_{n}^{0}=\emptyset$ and $L_{n} \subset B_{n}$,

- $\mu\left(D_{n} \backslash L_{n}\right)<1 / 2^{n}$ for all $n$, because $D_{n} \backslash L_{n} \subset U_{n}$,

- the function $f: L_{n} \rightarrow \mathbb{K}$ is continuous, because $L_{n} \subset C_{n}$ and $h_{n}=f$ on $C_{n}$; and

- their complements $X \backslash L_{n}$ are connected; indeed, this is trivial if $X \backslash$ $D_{n}$ is connected; if this would be not connected, then we would have $X \backslash L_{n}=\left(X \backslash D_{n}\right) \cup U_{n}=U_{n} \cup \bigcup_{j \in I(n)} E_{n, j}$ and the sets $U_{n}, E_{n, j}$ would be connected and each set $E_{n, j}$ shares at least a common point with $U_{n}$.

We consider now the measurable set $M:=\liminf _{n \rightarrow \infty} L_{n}=\bigcup_{n=1}^{\infty} \bigcap_{j=n}^{\infty} L_{j}$. By the subadditivity property of measures,

$$
\begin{aligned}
\mu\left(A_{n} \backslash L_{n}\right) \leq & \mu\left(A_{n} \backslash B_{n}\right)+\mu\left(B_{n} \backslash L_{n}\right) \\
& \leq \mu(D)+\mu\left(B_{n} \backslash C_{n}\right)+\mu\left(C_{n} \backslash D_{n}\right)+\mu\left(D_{n} \backslash L_{n}\right) \\
& \leq 0+\frac{1}{2^{n}}+\frac{1}{2^{n}}+\frac{1}{2^{n}}=\frac{3}{2^{n}} \quad(n \in \mathbb{N}) .
\end{aligned}
$$

¿From this and the facts that $M \supset \bigcap_{j=n}^{\infty} L_{j}$ for all $n$ and that the sequence $\left\{A_{n}\right\}_{n=1}^{\infty}$ is increasing, we derive

$$
\begin{aligned}
\mu\left(A_{n} \backslash M\right) & \leq \mu\left(A_{n} \backslash \bigcap_{j=n}^{\infty} L_{j}\right)=\mu\left(\bigcup_{j=n}^{\infty}\left(A_{n} \backslash L_{j}\right)\right) \\
& \leq \mu\left(\bigcup_{j=n}^{\infty}\left(A_{j} \backslash L_{j}\right)\right) \leq \sum_{j=n}^{\infty} \mu\left(A_{j} \backslash L_{j}\right)<\frac{6}{2^{n}} \quad(n \in \mathbb{N}) .
\end{aligned}
$$

Hence we get

$$
\mu(Y \backslash M)=\mu\left(\bigcup_{n=1}^{\infty}\left(A_{n} \backslash M\right)\right)=\lim _{n \rightarrow \infty} \mu\left(A_{n} \backslash M\right)=0,
$$


where the second equality is true because the sequence $\left\{A_{n} \backslash M\right\}_{n=1}^{\infty}$ is also increasing.

Finally, hypothesis (e) comes in our help, yielding the existence of a sequence $\left\{f_{n}\right\}_{n=1}^{\infty} \subset \mathcal{A}$ for which

$$
\left|f_{n}(x)-f(x)\right|<\frac{1}{n} \quad\left(x \in L_{n}, n \in \mathbb{N}\right) .
$$

Thus, it is enough to show that $f_{n}(x) \rightarrow f(x)(n \rightarrow \infty)$ whenever $x \in M$. For this, fix a point $x \in M$. Then a positive integer $N$ can be picked in such a way that $x \in L_{n}$ for all $n \geq N$, so $\left|f_{n}(x)-f(x)\right|<1 / n$ for the same integers $n$. This yields the desired result.

As an easy (and expected) example, note that if $X=\mathbb{R}^{N}(N \in \mathbb{N})$ with the usual topology, $\Sigma$ is the $\sigma$-algebra $\mathcal{L}_{N}$ of Lebesgue-measurable sets in $\mathbb{R}^{N}$ and $\mu=\lambda_{N}$ is the Lebesgue $N$-dimensional measure, then all hypotheses (a)-(d) in the last lemma are fulfilled.

\section{Approximation of measurable functions}

Here we apply the results of Sections 3-4 to obtain the following statement about universal measurable approximation.

Theorem 5.1. Let be prescribed $O, F, \varphi,\left\{P_{n}\right\}$ as in Theorem 3.1. Let us consider the sequence $\left\{\tau_{n}\right\}$ constructed in that theorem. Then $\left\{\tau_{n}\right\}$ enjoys the following approximation property: If a Lebesgue-measurable set $S \subset F$ and a complex Lebesgue-measurable function $f: S \rightarrow \mathbb{C}$ are given, then there exists a sequence $\left\{n_{k}\right\}$ of natural numbers such that

$$
\tau_{n_{k}}(z) \rightarrow f(z) \text { almost everywhere on } S \text {. }
$$

Proof. The unique task is to select a good "casting of characters" in Lemma 4.1 and to apply Theorem 3.1. For this, let us set $X=\mathbb{C}=\mathbb{R}^{2}$ with the usual topology, $\Sigma=\mathcal{L}_{2}, \mu=\lambda_{2}, \mathbb{K}=\mathbb{C}, Y=F \backslash\{1\}, \mathcal{A}=\left\{\tau_{n}: n \geq 1\right\}$. As noticed before, hypotheses (a)-(d) in Lemma 4.1 are fulfilled. As for hypothesis (e), observe that if $L \subset F \backslash\{1\}$ is a compact subset with empty interior and connected complement, then by Theorem $3.1 \mathcal{A}$ is dense in $A(L)=C(L)$. Since $\lambda_{2}(\{1\})=0$, we may assume without loss of generality that $S \subset F \backslash\{1\}$. Moreover, the function $f$ can be extended as a measurable 
function $f: Y \rightarrow \mathbb{C}$ just by setting $f(z)=0$ for all $z \in Y \backslash S$. Consequently, $f$ can be approximated almost everywhere on $Y$ (so on $S$ ) by a sequence of members of $\left\{\tau_{n}\right\}$, but this is exactly what is desired.

Several theorems, of the kind of Theorem 5.1, could be proved for universal Taylor series or other kinds of universal functions, for instance those studied in [10].

As remarked earlier, Theorem 1 of [1] is the special case $[O=\mathbb{D}, F=$ $\left.\mathbb{D}^{c}, \varphi=0, K \subset \partial \mathbb{D} \backslash\{1\}\right]$ of Theorem 3.1. The notation for $P_{n}, p_{n}, m_{n}, \tau_{n}, \sigma_{n}$ $(n \in \mathbb{N})$ that we used in [1] is similar to that of the present paper. We point out that in Theorems 2-3 of [1] we derived from Theorem 1 the following result on universal approximation of measurable functions by sequences of trigonometric polynomials, this time in the setting of $\lambda_{1}$ :

If $\left\{P_{n}\right\},\left\{p_{n}\right\},\left\{m_{n}\right\}$ are as in Theorem 1 and $g:[0,2 \pi] \rightarrow \mathbb{C}$ is a given Lebesgue-measurable complex function on $[0,2 \pi]$, then there are sequences $\left\{n_{k}\right\}$ and $\left\{l_{k}\right\}$ of natural numbers satisfying

$$
\begin{aligned}
& \alpha_{n_{k}}(t) \rightarrow g(t) \\
& \beta_{l_{k}}(t) \rightarrow g(t)
\end{aligned} \quad(k \rightarrow \infty) \text { almost everywhere on }[0,2 \pi],
$$

where

$$
\alpha_{n}(t)=\frac{1}{P_{n}} \sum_{\nu=0}^{m_{n}} p_{\nu}(\cos \nu t+i \sin \nu t)
$$

and

$$
\beta_{n}(t)=\frac{1}{P_{n}} \sum_{\nu=0}^{m_{n}} p_{\nu} \sum_{\mu=0}^{\nu}(\cos \mu t+i \sin \mu t) .
$$

A few results, similar to the just mentioned one, have already appeared in [10] and [13].

\section{ACKNOWLEDGEMENTS}

The authors are grateful to the referee for interesting and fruitful comments and suggestions. 


\section{References}

[1] L. Bernal-González, M.C. Calderón-Moreno and W. Luh, Universality and summability of trigonometric polynomials and trigonometric series, to appear in Periodica Math. Hungar.

[2] K. Faulstich, Summierbarkeit von Potenzreihen durch Riesz-Verfahren mit komplexen Erzeugendenfolgen, Mitt. Math. Sem. Gießen 139 (1979).

[3] K. Faulstich, W. Luh and L. Tomm, Universelle Approximation durch Riesz-Transformierte der geometrischen Reihe, Manuscripta Math. 36 (1981), 309-321.

[4] D. Gaier, "Lectures on Complex Approximation", Birkhäuser, Basel/London/Stuttgart, 1987.

[5] K. G. Große-Erdmann, Holomorphe Monster und Universelle Funktionen, Mitt. Math. Sem Gießen 176 (1987).

[6] J.P. Kahane, Baire's category theorem and trigonometric series, J. Analyse Math. 80 (2000), 143-182.

[7] L. Landau, D. Gaier, "Darstellung und Begründung einiger neuerer Ergebnisse der Funktionentheorie", Springer, Berlin/Heidelberg/New York/London/Paris/Tokyo, 1986.

[8] W. Luh, Kompakte Summierbarkeit von Potenzreihen im Einheitskreis, Acta Math. Acad. Sci. Hungar. 28 (1976), 51-54.

[9] W. Luh, Universal approximation properties of overconvergent power series, Analysis 6 (1986), 191-207.

[10] A. Melas and V. Nestoridis, Universality of Taylor series as a generic property of holomorphic functions, Adv. Math. 157 (2001), 138-176.

[11] A. Melas and V. Nestoridis, On various types of universal Taylor Series, Complex Variables Theory Appl. 44 (2001), 245-258.

[12] D. E. Mensǒv, Sur le séries trigonométrique universelles, Dokl. Acad. Sci. SSSR (N.S.), 49 (1945), 79-92. 
[13] V. Nestoridis, Universal Taylor Series, Ann. Inst. Fourier 46 (1996), 1293-1306.

[14] O.A. Nielsen, "An Introduction to Integration and Measure Theory", J. Wiley and Sons, Inc., New York, 1997.

[15] A. Peyerimhoff, "Lectures on summability", Lecture Notes in Mathematics, Springer, Berlin-Heidelberg-New York-Tokyo, 1970.

[16] W. Rudin, "Real and Complex Analysis", 3rd ed., McGraw-Hill, New York, 1987.

[17] A. Wilansky, "Summability through Functional Analysis", NordHolland, Amsterdam, 1984.

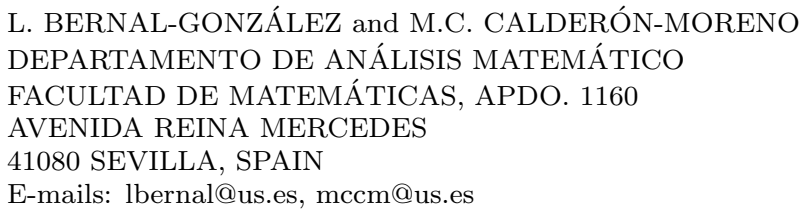

E-mails: lbernal@us.es, mccm@us.es

WOLFGANG LUH UNIVERSITÄT TRIER FACHBEREICH MATHEMATIK D-54286 TRIER GERMANY E-mail: luh@uni-trier.de 